\title{
Pertussis in New South Wales, 1993-2005: the impact of vaccination policy on pertussis epidemiology
}

\author{
Kerri A. Viney ${ }^{\mathrm{A}, \mathrm{C}}$, Jeremy M. McAnulty ${ }^{\mathrm{A}}$ \\ and Sue Campbell-Lloyd ${ }^{\mathrm{B}}$ \\ ${ }^{\mathrm{A} C o m m u n i c a b l e ~ D i s e a s e s ~ B r a n c h, ~ N S W ~ D e p a r t m e n t ~ o f ~ H e a l t h . ~}$ \\ ${ }^{\mathrm{B}}$ AIDS/Infectious Diseases Branch, NSW Department of Health. \\ ${ }^{\mathrm{C}}$ Corresponding author.Email: kvine@doh.health.nsw.gov.au
}

\begin{abstract}
Objective: To assess whether changes in vaccination policy have affected the epidemiology of pertussis in NSW between 1993 and 2005. Methods: Surveillance data from the NSW Notifiable Diseases Database was reviewed for the period. Results: 35695 cases of pertussis were notified; annual incidence rates varied from 18.4 to 84.2 per 100000 people. The highest rates of pertussis were consistently found in infants aged 0-6 months. Rates of disease in other age groups changed markedly over the study period, with high rates currently observed in adult age groups. Conclusions: New strategies may be needed to control pertussis in infants and in adults who now comprise the largest proportion of cases.
\end{abstract}

Pertussis, or whooping cough, is a highly contagious disease caused by the bacteria Bordetella pertussis. The disease is characterised by paroxysmal cough, inspiratory whoop and post-tussive vomiting. ${ }^{1}$ Pertussis is transmitted via direct contact with discharges from the respiratory tract of infected individuals (probably via droplets), and the incubation period is $9-10$ days on average. ${ }^{2} \mathrm{~A}$ secondary attack rate of $90 \%$ has been recorded in non-immune household contacts. ${ }^{2}$ Worldwide, the incidence of pertussis has been reported to be highest in children under 5 years, except where infant vaccination programs have achieved consistently high vaccination rates. ${ }^{2}$

In NSW, there have been several changes to the immunisation schedule affecting pertussis vaccination in recent years. From 1985, a combined whole cell diphtheria, tetanus and pertussis vaccine (DTPw) was included in the immunisation schedule at ages 2, 4 and 6 months, with a booster at 18 months. ${ }^{3}$ In 1994, a fifth dose was introduced into the schedule, which was given before school entry (at 4-5 years). In 1997, the fourth and fifth doses of DTPw were replaced by an acellular formulation (DTPa diphtheria, tetanus, acellular pertussis) and in 1999, all five doses were given as DTPa. ${ }^{4}$ In 2004, an adolescent booster vaccine (dTpa - diphtheria, tetanus, acellular pertussis) was introduced for 15-17 year olds and in NSW this was offered to all 11-18 year olds as part of the school based immunisation program. Also in 2004, the booster for 18 month olds was no longer recommended. ${ }^{3}$

Under the NSW Public Health Act 1991, cases of pertussis are notified by doctors, hospitals, laboratories, schools and childcare facilities. ${ }^{5}$ Prior to January 2006 public health unit staff routinely followed up all reported cases of pertussis; since January 2006 only cases aged less than 20 years are investigated.

We conducted a descriptive study to assess the impact of changes to vaccination policy on pertussis epidemiology in NSW.

\section{Methods}

We reviewed surveillance data from the NSW Notifiable Diseases Database (NDD) for the period 1 January 1993 to 31 December 2005. For our review, a case of pertussis was defined according to the national case definition. ${ }^{6}$

Annual notification rates were calculated using mid year population estimates from the Australian Bureau of Statistics. Notification rates and frequencies were calculated using SAS (version 8.2 SAS Institute, Cary, NC, USA). Cases were analysed by date of onset, method of diagnosis, area of residence, age group, gender and mortality. Data on mortality were taken from NDD. Age groups analysed were: 0-6 months, 7-11 months, 1-4 years, 5-11 years, 12-17 years, 18-24 years, 25-44 years, 45-64 years and 65 years and older. Age groups were chosen to reflect ages at which vaccination occurs and stages of adulthood.

To assess the impact of changes in vaccination policy on age specific disease incidence, the above case characteristics were compared over 5 time periods:

(1) before the introduction of the fifth dose (1993-94)

(2) after the introduction of the fifth dose (1995-98) 
(3) after the introduction of acellular vaccines for all five doses (1999-2003)

(4) during the school-based adolescent booster campaign (2004) and

(5) after the introduction of the adolescent booster (2005).

\section{Results}

From 1 January 1993 to 31 December 2005, a total of 35695 cases of pertussis were reported in NSW (an average of 2746 cases each year). Fig. 1 displays the number of notifications during the study period; notifications peaked seven times, in November 1993, November 1997, September 2000, August 2001, November 2003, September 2004, and in August 2005.

Annual incidence rates per 100000 people ranged from a low of 18.4 in 1996 to a high of 84.2 in 2005. The overall incidence rate over the 13 years was 42.8 per 100000 people. Incidence rates varied widely between Area Health Service of residence and by year of notification. Metropolitan Sydney had an incidence rate of 39.8 per 100000 people, compared with 47.1 for the rest of NSW. Of all notified cases, females comprised $56.1 \%$ and males $43.7 \%$. There was a slight increase in the proportion of female cases reported over time, and in 2005 this reached its highest at $60 \%$.

Table 1 displays the number of pertussis cases and incidence for the five periods studied, and also provides these figures by gender, age group, method of diagnosis, location of residence and number of deaths. Fig. 2 displays the incidence rates of pertussis per 100000 people for six age groups by the study periods, and Fig. 3 displays the proportion of pertussis cases by age group and study period. These results are further described below.

\section{1993-94}

In the 1993-94 period, 2938 pertussis cases were notified (incidence rate, 24/100000). The highest notification rates were in infants aged $0-6$ months followed by infants aged 7-11 months and children aged 5-11 years. The lowest notification rates were in those aged 65 years and older. The predominant method of diagnosis was serology. No deaths were recorded during this period.

\section{1995-98}

Between 1995 and 1998, 9078 cases of pertussis were notified (incidence rate, 35.9/100000). The highest notification rates were in infants aged 0-6 months, followed by primary school age children (5-11 years), and adolescents aged 12-17 years. Compared with 1993-94, rates in all adult age groups increased, rates in school age children increased (by approximately 50\%) and rates in infants aged 7-11 months decreased. Rates in children aged 1-4 years old showed almost no change. In 1995-98 the predominant method of diagnosis was serology. Five deaths were recorded in infants aged less than 12 months of age, two of whom were less than 1 month old. ${ }^{7}$

\section{1999-2003}

Between 1999 and 2003, 14317 cases of pertussis were notified (incidence rate, 43.1/100000). The highest rates

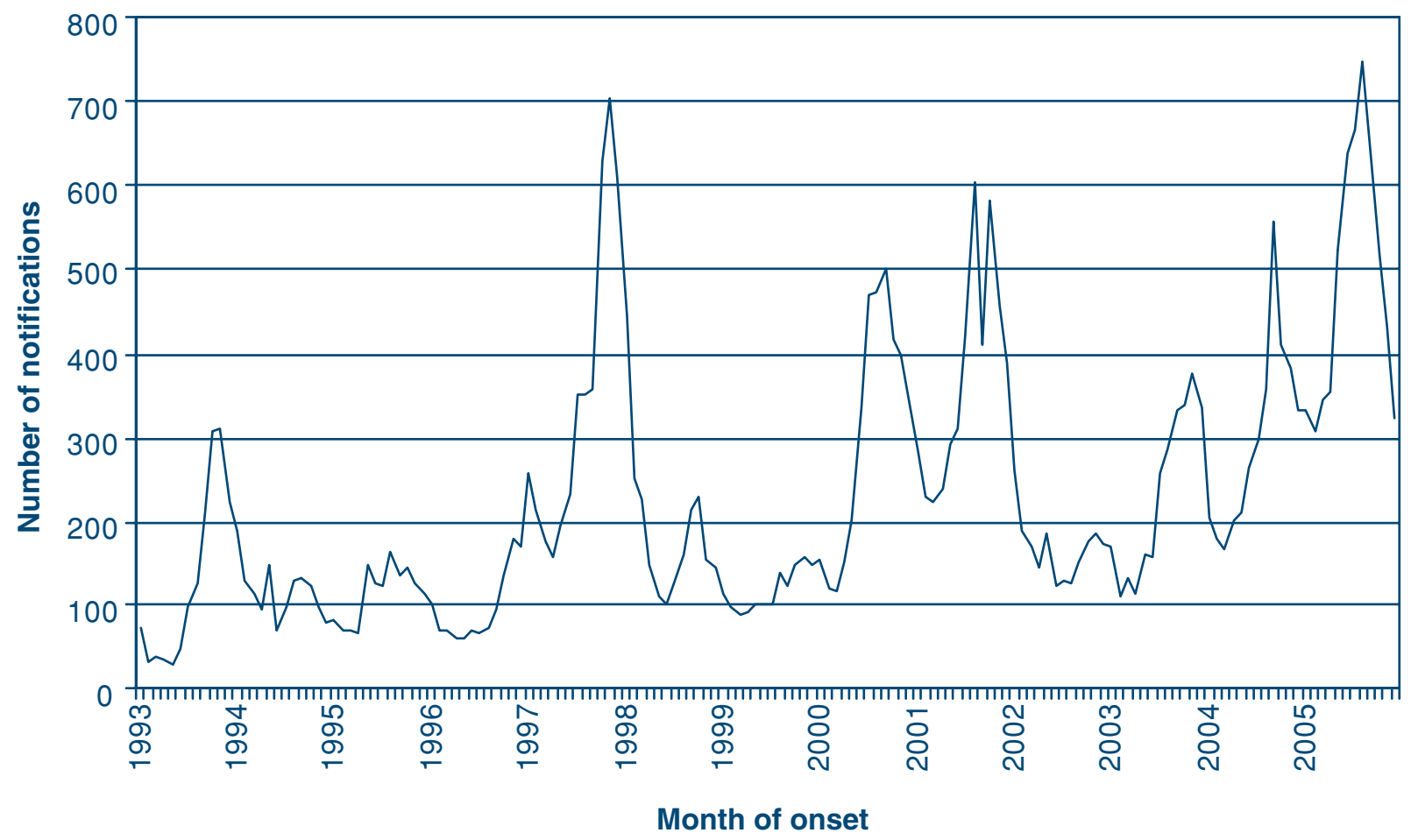

Fig. 1. Number of pertussis notifications by month of onset, NSW, 1993-2005. 
were in infants aged between 0-6 months, followed by adolescents and primary school age children. Compared with 1995-98, rates in children aged 5-11 years decreased by $38 \%$. Rates in children aged between $1-4$ years and in infants aged between 0-6 months also decreased, and rates in cases aged over 11 years increased, mainly in 12-17 year olds (by 109\%). Two-thirds of cases were diagnosed by serology and $12 \%$ were diagnosed by both polymerase chain reaction (PCR) and culture (PCR was introduced as a diagnostic method in 1999). Three deaths were recorded; one in a 2-month-old infant and two in adults aged 75 and 78 years.

\section{2004}

In 2004, there were 3563 cases of pertussis (incidence rate, 52.3/100000). The highest rates were in infants aged 0-6 months, followed by adolescents and adults aged 45-64 years (for the first time, rates in an adult age group became the third highest). Rates in other adult age groups also increased, most notably in adults aged 65 years and over, where rates increased by $134 \%$. Just over threequarters of notifications were diagnosed by serology and
$13 \%$ by PCR. There was one death due to pertussis in a 95-year-old person.

\subsection{5}

In 2005,5799 cases of pertussis were notified (incidence rate, 84.2/100000). Highest notification rates were in infants aged 0-6 months, followed by adults aged 45-64 years and adults aged 25-44 years. Compared to 2004, rates decreased by over $50 \%$ in adolescents and by $32 \%$ in children aged 5-11 years. Rates increased for all adult age groups and for children under 1 year of age, when compared to 2004. In 2005, almost all cases (85\%) were diagnosed by serology while $7 \%$ were diagnosed by PCR. No deaths were recorded in 2005 .

\section{Discussion}

The age distribution of pertussis notifications in NSW changed over the study period. In 1993-94, approximately one-third of cases occurred in adults, by 2005 this had increased to almost $90 \%$ (Fig. 3). The incidence of pertussis in children aged less than 12 years declined, except for $0-6$ month olds where there was a slight increase

Table 1. Characteristics of pertussis cases in NSW, 1993-2005, by study periods

\begin{tabular}{|c|c|c|c|c|c|c|c|c|c|c|c|c|c|c|c|}
\hline & \multicolumn{3}{|c|}{ 1993-94 } & \multicolumn{3}{|c|}{ 1995-98 } & \multicolumn{3}{|c|}{ 1999-2003 } & \multicolumn{3}{|c|}{2004} & \multicolumn{3}{|c|}{2005} \\
\hline & $n$ & $\%$ & $\begin{array}{c}\text { Rate/ } \\
100000\end{array}$ & $n$ & $\%$ & $\begin{array}{l}\text { Rate/ } \\
100000\end{array}$ & $n$ & $\%$ & $\begin{array}{l}\text { Rate/ } \\
100000\end{array}$ & $n$ & $\%$ & $\begin{array}{c}\text { Rate/ } \\
100000\end{array}$ & $n$ & $\%$ & $\begin{array}{c}\text { Rate/ } \\
100000\end{array}$ \\
\hline \multicolumn{16}{|l|}{ Gender } \\
\hline Male & 1362 & 46.4 & 22.7 & 4089 & 45.1 & 33 & 6360 & 44.4 & 39.1 & 1500 & 42.1 & 44.8 & 2295 & 39.6 & 67.8 \\
\hline Female & 1572 & 53.5 & 25.9 & 4942 & 54.7 & 39.6 & 7955 & 55.6 & 48.2 & 2061 & 57.8 & 60.9 & 3496 & 60.3 & 102.3 \\
\hline Unknown & 4 & 0.1 & - & 20 & 0.2 & - & 2 & 0 & - & 2 & 0.1 & - & 8 & 0.1 & - \\
\hline \multicolumn{16}{|l|}{ Age group ${ }^{A}$} \\
\hline $0-6$ months & 183 & 6.2 & 208.1 & 411 & 4.5 & 237.3 & 464 & 3.2 & 216.5 & 71 & 2 & 164.4 & 94 & 1.6 & 218.1 \\
\hline 7-11 months & 65 & 2.2 & 73.9 & 69 & 0.8 & 39.8 & 88 & 0.6 & 41.1 & 16 & 0.4 & 37.1 & 22 & 0.4 & 51 \\
\hline $1-4$ years & 347 & 11.8 & 49.3 & 694 & 7.6 & 49.2 & 647 & 4.5 & 37.1 & 157 & 4.4 & 46.2 & 152 & 2.6 & 44.8 \\
\hline 5-11 years & 884 & 30.1 & 73.6 & 2670 & 29.4 & 108.6 & 2139 & 14.9 & 67.6 & 205 & 5.8 & 32.9 & 141 & 2.4 & 22.5 \\
\hline $12-17$ years & 379 & 12.9 & 37.7 & 1225 & 13.5 & 59.3 & 3324 & 23.2 & 124 & 503 & 14.1 & 92 & 223 & 3.8 & 40.4 \\
\hline $18-24$ years & 133 & 4.5 & 10.2 & 479 & 5.3 & 19.2 & 799 & 5.6 & 26 & 236 & 6.6 & 36.7 & 423 & 7.3 & 65.8 \\
\hline $25-44$ years & 568 & 19.1 & 15.3 & 2007 & 22.1 & 26.2 & 3471 & 24.2 & 35.2 & 970 & 27.2 & 49.2 & 1900 & 32.8 & 96.1 \\
\hline 45-64 years & 267 & 9.1 & 10.8 & 1134 & 12.5 & 21.2 & 2614 & 18.3 & 34.6 & 1023 & 28.7 & 63.4 & 2080 & 35.9 & 125.4 \\
\hline $65+$ years & 112 & 3.8 & 7.5 & 389 & 4.3 & 12.3 & 770 & 5.4 & 18 & 382 & 10.7 & 42.1 & 764 & 13.2 & 82.9 \\
\hline \multicolumn{16}{|l|}{ Method of diagnosis } \\
\hline Serology & 1364 & 46.4 & - & 5533 & 60.9 & - & 9541 & 66.6 & - & 2728 & 76.6 & - & 4952 & 85.4 & - \\
\hline PCR & 0 & 0 & - & 0 & 0 & - & 1747 & 12.2 & - & 478 & 13.4 & - & 405 & 7 & - \\
\hline Clinical & 30 & 1 & - & 556 & 6.1 & - & 1755 & 12.3 & - & 227 & 6.4 & - & 182 & 3.1 & - \\
\hline Culture & 183 & 6.2 & - & 339 & 3.7 & - & 260 & 1.8 & - & 21 & 0.6 & - & 23 & 0.4 & - \\
\hline Other/unknown & 1361 & 46.4 & - & 2650 & 29.3 & - & 1014 & 7.1 & - & 109 & 3.1 & - & 237 & 4.1 & - \\
\hline \multicolumn{16}{|l|}{ Residence } \\
\hline Metro Sydney & 1602 & 54.5 & 22.7 & 4866 & 53.6 & 33.2 & 7158 & 50 & 37.1 & 2109 & 59.2 & 53.1 & 3791 & 65.4 & 94.3 \\
\hline Other NSW & 1336 & 45.5 & 26.7 & 4211 & 46.4 & 41 & 7159 & 50 & 53.2 & 1454 & 40.8 & 52.7 & 2008 & 34.6 & 72.1 \\
\hline Deaths & 0 & 0 & 0 & 5 & 0.05 & 0.02 & 3 & 0.02 & 0 & 1 & 0.03 & 0 & 0 & 0 & 0 \\
\hline Total & 2938 & & & 9078 & & & 14317 & & & 3563 & & & 5799 & & \\
\hline
\end{tabular}

AOne person in 1999-2003 had age group missing. 
(Fig. 2). This increase occurred despite the introduction of a fifth dose, an adolescent booster vaccination and the introduction of acellular vaccinations.

Infants aged 0-6 months are at greatest risk of pertussis because they are partially immunised; for example, the effectiveness of acellular pertussis vaccines in preventing hospitalisation of children aged 2-32 months has been demonstrated to be $68.1 \%$ after one dose of vaccine, $91.8 \%$ after the second dose, and $99.8 \%$ after three doses. ${ }^{8}$ In addition, the protective efficacy of three doses of acellular vaccine is approximately $84 \% .{ }^{9}$ Higher rates observed in infants in 2005 may reflect epidemic activity; pertussis epidemics typically occur every 3-4 years in NSW and the last recorded epidemic was between 2000-02. ${ }^{10}$ Higher rates in infants may also be due to infants being infected by older adults. Rates of pertussis have increased in all adult age groups aged 18 years and older. There was a decrease in rates in high school aged children (12-17 years) after the introduction of the adolescent school-based immunisation program in 2004.

In countries with high immunisation rates, a greater proportion of pertussis cases are now presenting in adults and adolescents. ${ }^{1,11,12,13}$ In the United States from 1994-96 to 1997-2000, incidence rates in adolescents and adults increased by $62 \%$ and $60 \%$, respectively. ${ }^{14}$ In Canada from 1993-98, the proportion of pertussis cases in adults steadily increased ${ }^{15}$ and in 2000 for the first time in two decades, 10-14-year-old children represented a larger pro- portion of cases (34\%) and had a higher incidence of pertussis than infants or preschool age children $(13 \%) .{ }^{15} \mathrm{In}$ Europe, recent pertussis infection was found to be significantly more likely in adults and adolescents in countries with high immunisation rates. ${ }^{12} \mathrm{~A}$ trend towards pertussis occurring in older age groups has also been documented in NSW in the Hunter/New England Area Health Service. ${ }^{16}$

Despite high vaccination rates in many countries, pertussis continues to circulate. ${ }^{20}$ Reasons for this include: waning immunity following both natural infection and immunisation, ${ }^{11,20,21,22}$ increased recognition of pertussis in adolescents by physicians, ${ }^{1}$ increased use of serology and PCR testing, ${ }^{11}$ improved surveillance, ${ }^{19}$ in some countries the use of a poorly protective vaccine resulting in cohorts of susceptible people, ${ }^{11,15}$ and of course, a real increase in pertussis. ${ }^{19,21}$ The relative contribution of these factors in NSW is unclear, but it is likely to be a combination of many of these factors. From 1995-2004, DTP vaccination rates at age $12-15$ months in NSW have ranged from $83.3 \%$ in 1998 to $92.5 \%$ in $2004 .^{23,24}$

The increase in pertussis in adolescents and adults in NSW is of concern because they may serve as reservoirs of infection for partially immunised infants. ${ }^{13,20,21,25}$ In studies from the United States, ${ }^{26}$ France, ${ }^{27}$ the United Kingdom ${ }^{28}$ and Australia ${ }^{29}$ parents were identified as the source of infection for $34-47 \%$ of infections in infants. Further, studies from several countries including Canada, Denmark, and France indicate that up to $32 \%$ of adults and

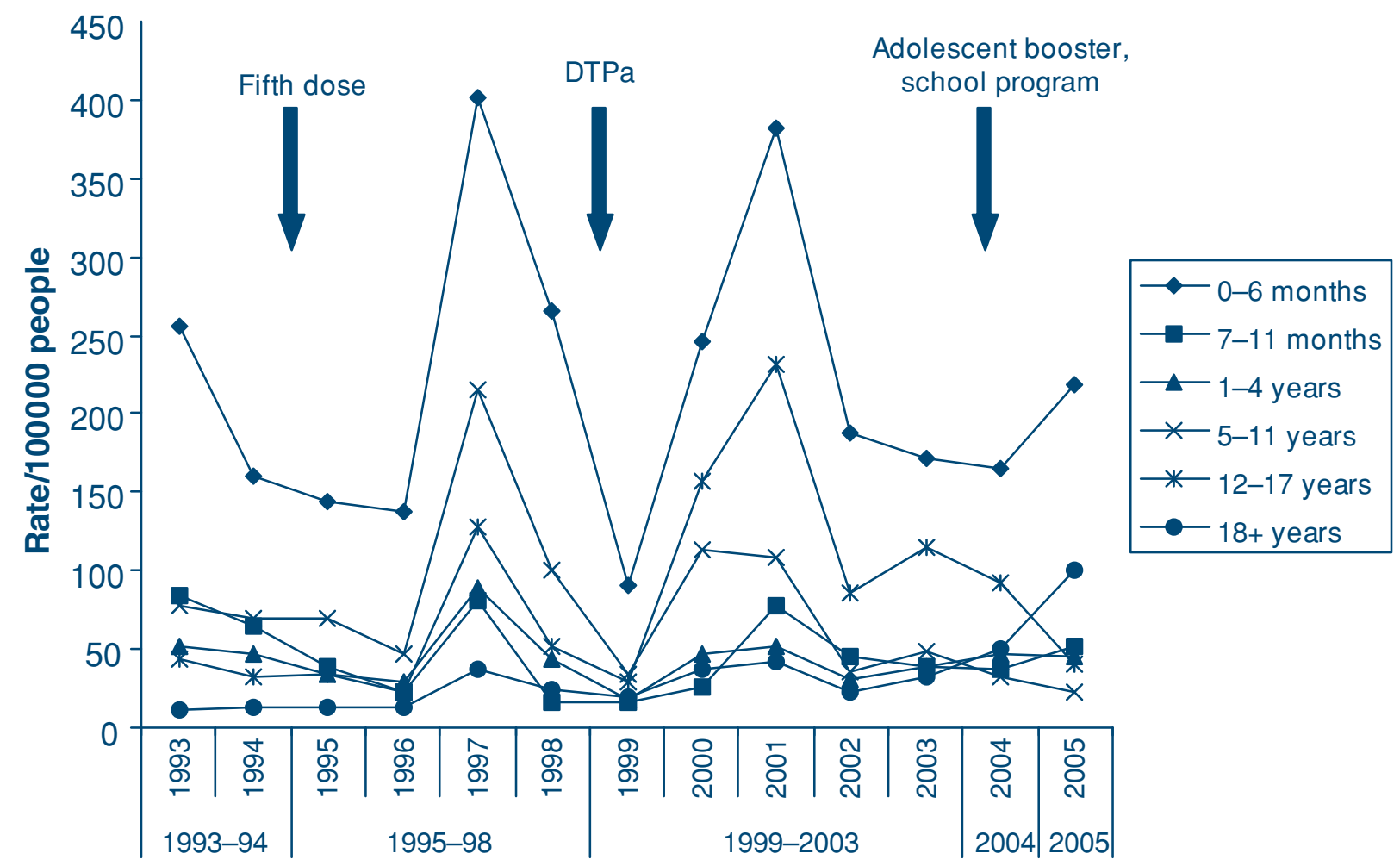

Fig. 2. Incidence rates of pertussis/100000 people by age group at onset and the introduction of changes to the vaccination schedule, NSW, 1993-2005. 
adolescents with a coughing illness of at least 1 week's duration have pertussis. ${ }^{30,31}$ This proportion may change depending on the nature of pertussis activity at the time; during pertussis epidemics this may be higher than at other times. Unpublished NDD data on $15 \mathrm{NSW}$ children aged under 12 months indicates that although for half the source of infection was unknown, an adult was identified as a potential source of infection in $13 \%$ of cases and young children in 33\%. No adolescent was identified as a source.

In recognition of the rising incidence of pertussis in adolescents and adults, and the role they may play in transmission to infants, ${ }^{17}$ Australia, Austria, Canada, France and Germany have incorporated an adolescent booster into their immunisation schedules, ${ }^{18}$ and it is now recommended in the USA. ${ }^{19}$

There are several limitations to this analysis. First, laboratory notification of pertussis began in 1991 and data quality may have improved over time, therefore later data may be more complete than earlier data. Second, following substantial increases in pertussis notifications based on serological testing in 2005, a review of the antibody test was carried out. ${ }^{32}$ The reviewers concluded that the use of the antibody test was likely to have resulted in over diag- nosis of pertussis. ${ }^{32}$ In $2005,85 \%$ of cases were diagnosed using serology and there may have been an over diagnosis of pertussis in this year, particularly in adults.

Serology as a diagnostic method is not recommended in children less than 2 years of age due to its low sensitivity in this age group. ${ }^{5}$ PCR, however, is largely replacing culture for the diagnosis of pertussis. ${ }^{5,33}$ Because PCR and serology are more likely than culture to be positive in both adults and older children with pertussis, the increased availability and use of PCR and serology may contribute to an increase in pertussis cases that may have otherwise been undiagnosed..$^{11,33}$

In NSW, pertussis may now be regarded as a disease primarily of adults although the severest outcomes remain for infants and potentially the elderly. One-third of cases in 2005 occurred in people of childbearing age, and this may pose a risk to unimmunised infants. Rates in adults increased 5-13 fold over the study period, and have increased the most in adults aged 45-64 years; this is of concern as these people may be providing childcare for children or grandchildren. A lack of natural boosting due to less exposure to pertussis may contribute towards waning immunity in adults. ${ }^{31}$ Females now comprise $60 \%$

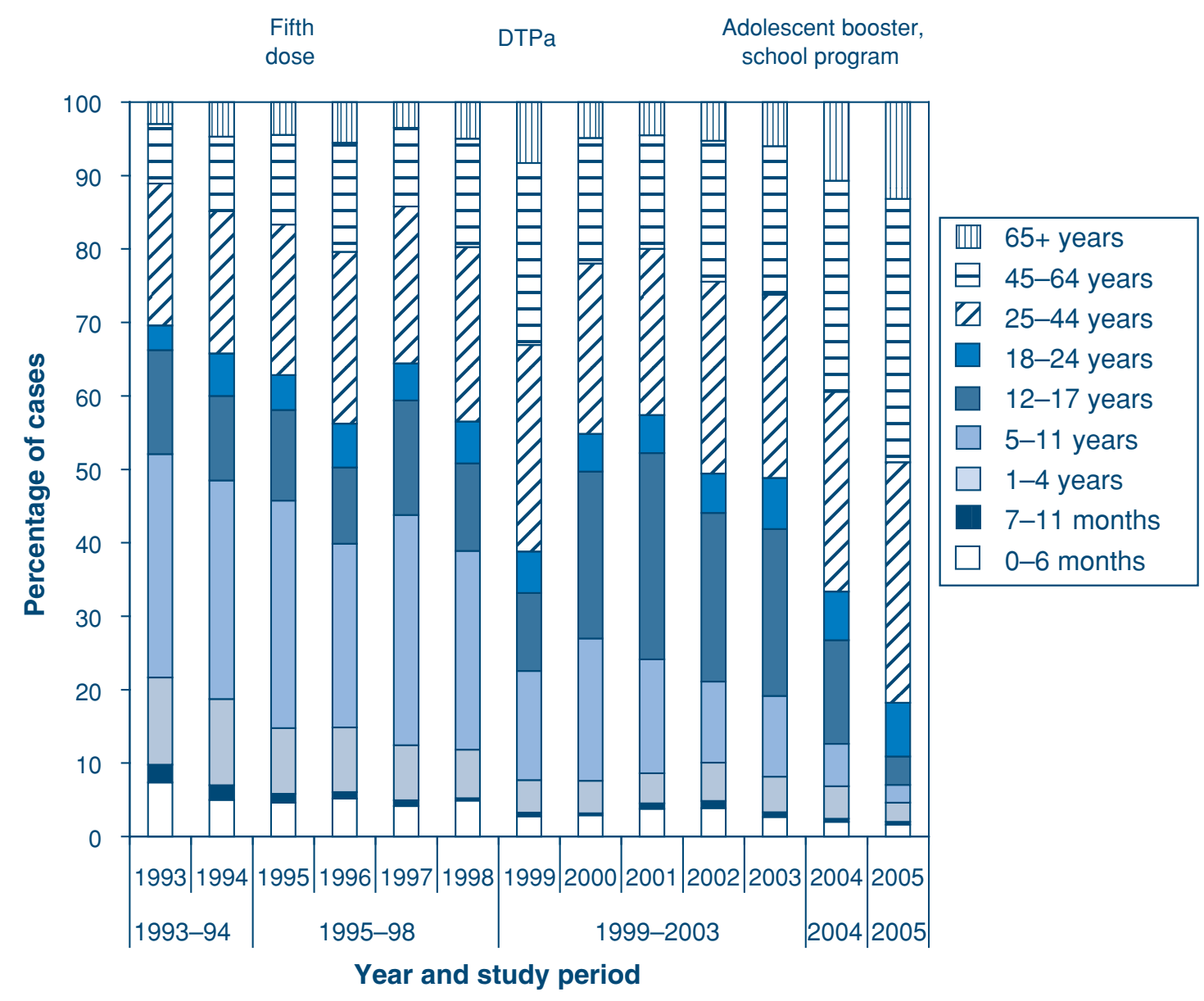

Fig. 3. Proportion of pertussis cases by age group at onset and study period in NSW, 1993-2005. 
of cases and, in their roles as mothers and grandmothers, may pose a risk to infants. Females also make up a large proportion of the health care and childcare workforce, thereby potentially posing an additional risk to infants in child care or health care settings. More data would be useful on the source of infection for infants aged less than 12 months in NSW to assess the impact of adolescent vaccination on those infants. In unpublished NDD data, the source of infection in over $50 \%$ of cases aged less than 12 months in 2006 was unknown.

The National Health and Medical Research Council recommends a booster vaccine for: adolescents; adults before planning pregnancy or as soon as possible after delivery of an infant; adults working with young children (especially for child care workers and health care workers); and adults who express an interest in receiving a booster dose of dTpa (provided that they have received a full course of DTP). ${ }^{34}$ Further, the NSW Department of Health policy states that all employees and other clinical personnel who have contact with clients should receive a dose of an acellular pertussis containing vaccine. ${ }^{35}$

\section{Conclusion}

Rates in infants aged less than 12 months, who are at greatest risk of severe disease and death, have not decreased over the thirteen year study period despite changes to the immunisation schedule. Other strategies may be required to effectively control pertussis in this age group. Vaccination of adolescents in the school based immunisation program may continue to influence rates of disease in infants, ${ }^{36}$ but strengthening of immunisation in new parents, ${ }^{37,38}$ child care and health care workers, ${ }^{37}$ adults planning a pregnancy ${ }^{37}$ as well as grand parents, should be considered further to both decrease rates of disease in infants and reduce morbidity in adults.

\section{References}

1. Centers for Disease Control and Prevention Pertussis-United States, 2001-2003. Morb Mortal Wkly Rep 2005; 54: 1283-6.

2. Heymann DL. Control of communicable diseases manual. 18th ed. Washington DC: American Public Health Association, 2004.

3. National Centre for Immunisation Research and Surveillance. A brief history of vaccination and childhood vaccination practices in Australia. Available at http://www.ncirs.usyd.edu.au/publ/publ-79-histtab1.html [Verified 15 May 2007].

4. Torvaldsen S, Simpson JM, McIntyre PB. Effectiveness of pertussis vaccinations in New South Wales, Australia, 1996-1998. Eur J Epidemiol 2003; 18: 63-9. doi:10.1023/A:1022588118030

5. Health NSW. Notifiable diseases manual. NSW: NSW Health, 2005.

6. Department of Health and Ageing. Australian national notifiable diseases case definitions-pertussis. Available at http://www.health.gov.au/internet/wcms/publishing.nsf/Content /cda-surveil-nndss-casedefs-cd_pertus.htm [Verified 15 May 2007].

7. National Centre for Immunisation Research and Surveillance Vaccine preventable diseases and vaccination coverage in Australia, 1993-1998. Commun Dis Intell 2000; 24: i-83.

8. Juretzko P, von Kries R, Wirsing von Konig CH, Weil J, Giani G. Effectiveness of acellular pertussis vaccine assessed by hospital-based active surveillance in Germany. Clin Infect Dis 2002; 35: 162-7. doi:10.1086/341027

9. Greco D, Salmaso S, Mastrantonio P, Giulianop M, Tozzi AE, Anemona A et al. A controlled trial of two acellular vaccines and one whole-cell vaccine against pertussis. $N$ Engl J Med 1996; 334: 341-8. doi:10.1056/NEJM199602083340601

10. Brotherton J, McAnulty J. A pertussis epidemic in NSW: how epidemiology reflects vaccination policy. N SW Public Health Bull 2003; 14: 77-81.

11. Skowronski D, De Serres G, MacDonald D, Wu W, Shaw C, Macnabb J et al. The changing age and seasonal profile of pertussis in Canada. J Infect Dis 2002; 185: 1448-53. doi: $10.1086 / 340280$

12. Pebody RG, Gay J, Giammanco A, Baron S, Schellekens J, Tischer A et al. The seroepidemiology of Bordetella pertussis infection in Western Europe. Epidemiol Infect 2005; 133(1): 159-71. doi:10.1017/S0950268804003012

13. Grimprel E, Baron S, Levy-Bruhl D, Garnier JM. N'jamkepo E, Guiso N, Begue P. Influence of vaccination coverage on pertussis transmission in France. Lancet 1999; 354: 1699-700. doi:10.1016/S0140-6736(99)01576-7

14. Centers for Disease Control and Prevention Pertussis-United States, 1997 - 2000. Morb Mortal Wkly Rep 2002; 51: 73-6.

15. Ntezayabo B, De Serres G, Duval B. Pertussis resurgence in Canada largely caused by a cohort effect. Pediatr Infect Dis $J$ 2003; 22: 22-7. doi:10.1097/00006454-200301000-00009

16. Durrheim D, Massey P, Carr C, Islam F. The changing epidemiology of pertussis in the Hunter New England Area and potential implications for the immunisation schedule. N S W Public Health Bull 2006; 17(3-4): 48-51.

17. Tan T. Summary: Epidemiology of pertussis. Pediatr Infect Dis J 2005; 24: S35-8. doi:10.1097/01.inf.0000160910. 17950.59

18. Tan T, Trinlade E, Skowronski D. Epidemiology of pertussis. Pediatr Infect Dis J 2005; 24: S10-8. doi:10.1097/01.inf. 0000160708.43944 .99

19. Centers for Disease Control and Prevention Preventing tetanus, diphtheria, and pertussis among adolescents: use of tetanus toxoid, reduced diphtheria toxoid and acellular pertussis vaccines. Morb Mortal Wkly Rep 2006; 55: 1-43.

20. Forsyth K, Tan T, Wirsing von Konig C-H, Caro JJ, Plotkin S. Potential strategies to reduce the burden of pertussis. Pediatr Infect Dis J 2005; 24: S69-74. doi:10.1097/01.inf.0000160917.29723.03

21. Schellekens J, Wirsing von Konig C-H, Gardner P. Pertussis sources of infection and routes of transmission in the vaccination era. Pediatr Infect Dis J 2005; 24: S19-24. doi:10.1097/01.inf.0000160909.24879.e6

22. Wendelboe AM, Van Rie A, Salmaso S, Englund JA. Duration of immunity against pertussis after natural infection or vaccination. Pediatr Infect Dis J 2005; 24: S58-61. doi:10.1097/01.inf.0000160914.59160.41 
23. Australian Bureau of Statistics. Children's immunisation Australia April 1995. Canberra: Australian Bureau of Statistics, 1996.

24. Medicare Australia. Australian childhood immunisation register. 1998-2004. Medicare Australia.

25. Greenberg DP. Pertussis in adolescents: increasing incidence brings attention to the need for booster immunization of adolescents. Pediatr Infect Dis $J$ 2005; 24: 721-8. doi:10.1097/01.inf.0000172905.08606.a3

26. Bisgard KM, Pascaul FB, Ehresmann KR, Miller CA, Cianfrani $\mathrm{C}$, Jennings $\mathrm{CE}$ et al. Infant pertussis: who was the source? Pediatr Infect Dis J 2004; 23: 985-9. doi:10.1097/01.inf.0000145263.37198.2b

27. Baron S, Njamkepo E, Grimprel E. Begue Ps, Desenclos J-C, Drucker J, Guiso N. Epidemiology of pertussis in French hospitals in 1993 and 1994: thirty years after a routine vaccination. Pediatr Infect Dis $J$ 1998; 17: 412-8. doi:10.1097/00006454-199805000-00013

28. Crowcroft NS, Booy R, Harrison T, Spicer L, Britta J, Mok Q et al. Severe and unrecognised: pertussis in UK infants. Arch Dis Child 2003; 88: 802-6. doi:10.1136/adc.88.9.802

29. Elliott E, McIntyre P, Ridley G, Morris A, Massie J, Mceniery J et al. National study on infants hospitalized with pertussis in the acellular vaccine era. Pediatr Infect Dis J 2004; 23: 246-52. doi:10.1097/01.inf.0000116023.56344.46

30. Rothstein E, Edwards E. Health burden of pertussis in adolescents and adults. Pediatr Infect Dis J 2005; 24: S44-7. doi:10.1097/01.inf.0000160912.58660.87
31. Hewlett EL, Edwards KM. Pertussis-not just for kids. $N$ Engl J Med 2005; 352: 1215-22. doi:10.1056/NEJMcp041025

32. Hueston L, Lanser J, Gidding H, Gilbert L. False positive pertussis tests. Available at http://www.health.nsw.gov.au/ infect/pdf/false_pertussis_tests.pdf [Verified 15 May 2007].

33. Halperin S. The control of pertussis-2007 and beyond. $N$ Engl J Med 2007; 356(2): 110-2. doi:10.1056/NEJMp068288

34. National Health and Medical Research Council. The Australian immunisation handbook. 8th ed. Canberra: Australian Government Department of Health and Ageing, 2003.

35. NSW Health. Policy directive: occupational assessment, screening and vaccination against specified infectious diseases. NSW: NSW Health, 2007.

36. Hethcote HW, Horby P, McIntyre P. Using computer simulations to compare pertussis vaccination strategies in Australia. Vaccine 2004; 22: 2181-91. doi:10.1016/j.vaccine.2003.11.053

37. Forsyth K, Nagai M, Lepetic A, Trindale E. Pertussis immunization in the Global Pertussis Initiative International Region: Recommended strategies and implementation considerations. Pediatr Infect Dis J 2005; 24: S93-7. doi:10.1097/01.inf. 0000160921.74004 .12

38. Scuffman PA, McIntyre PB. Pertussis vaccination strategies for neonates - an exploratory cost-effectiveness analysis. Vaccine 2004; 22: 2953-64. doi:10.1016/j.vaccine.2003.11.057

\section{A Decisive Decade in Immunisation}

The National Centre for Immunisation Research \& Surveillance (NCIRS) is pleased to announce its 10th Anniversary, which will be celebrated with a 1-day conference on Wednesday 18 July 2007 titled 'A Decisive Decade in Immunisation. This will be followed by a dinner.

The conference will be held in conjunction with the 2nd National Immunisation Workshop and will feature prominent international guest speakers including Professor Scott Halperin from the Canadian Center for Vaccinology and Professor Heinz-Josef Schmitt from Johannes Gutenberg University, Germany.

The venue is The Refectory, The University of Sydney. Details regarding these events including programs for both days and registration forms can be found at http://www.ncirs.usyd.edu.au/ or call or email Joanne Perkins (Ph:(02) 9845 1433, Email:joannep3@chw.edu.au) for further information. 